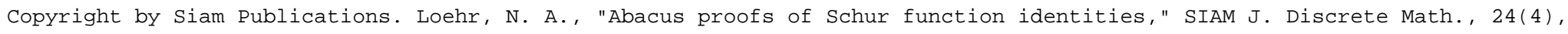
1356-1370, (2010). DOI: 10.1137/090753462

\title{
ABACUS PROOFS OF SCHUR FUNCTION IDENTITIES*
}

\author{
NICHOLAS A. LOEHR ${ }^{\dagger}$
}

\begin{abstract}
This article uses combinatorial objects called labeled abaci to give direct combinatorial proofs of many familiar facts about Schur polynomials. We use abaci to prove the Pieri rules, the Littlewood-Richardson rule, the equivalence of the tableau definition and the determinant definition of Schur polynomials, and the combinatorial interpretation of the inverse Kostka matrix (first given by Eğecioğlu and Remmel). The basic idea is to regard formulas involving Schur polynomials as encoding bead motions on abaci. The proofs of the results just mentioned all turn out to be manifestations of a single underlying theme: when beads bump, objects cancel.
\end{abstract}

Key words. abaci, Schur functions, Pieri rules, Littlewood-Richardson rules, symmetric polynomials, tableaux, inverse Kostka matrix

AMS subject classifications. 05E05, 05A19

DOI. $10.1137 / 090753462$

1. Introduction. An abacus is a combinatorial object that is useful for proving certain facts about integer partitions. Formally, an unlabeled abacus with $N$ beads is a word $w=\left(w_{k}: k \geq 0\right)$ such that each $w_{k}$ is 0 or 1 , and there are exactly $N$ indices $k$ with $w_{k}=1$. Here 1 represents a bead on the abacus, and 0 represents a gap. James and Kerber [7] used abaci to establish basic facts about $k$-cores and $k$-quotients of partitions. Abaci can also be used to prove the famed Jacobi triple product identity [3].

In this paper, we study objects called labeled abaci, which are combinatorial models for certain antisymmetric polynomials. We use these objects to provide direct combinatorial proofs of many familiar identities involving Schur polynomials. In particular, we use abaci to prove the following results:

- the Pieri rules, which describe the Schur expansion of the product of a Schur polynomial $s_{\lambda}$ by either a power-sum symmetric function $p_{k}$, a homogeneous symmetric function $h_{k}$, or an elementary symmetric function $e_{k}$;

- the equivalence of the combinatorial definition of Schur polynomials (in terms of semistandard tableaux) and the algebraic definition of Schur polynomials (as quotients of two determinants);

- the Littlewood-Richardson rule, which describes the Schur expansion of skew Schur polynomials and products of Schur polynomials;

- Eğecioğlu and Remmel's combinatorial interpretation of the inverse Kostka matrix $[1,5]$, which involves special rim-hook tableaux;

- a Pieri-type result for computing the Schur expansion of the product of $s_{\nu}$ and a monomial symmetric polynomial $m_{\lambda}$.

Our combinatorial approach reveals striking similarities between the proofs of these seemingly disparate results. In every proof, we interpret the data on one side of a combinatorial identity as encoding a sequence of bead motions on an abacus. The recurring theme is that when beads bump, objects cancel; the remaining objects correspond to the expression on the other side of the desired identity. Our combinatorial

\footnotetext{
${ }^{*}$ Received by the editors March 23, 2009; accepted for publication August 25, 2010; published electronically October 14, 2010. This work was supported in part by NSA research grant H9823008-1-0045.

http://www.siam.org/journals/sidma/24-4/75346.html

${ }^{\dagger}$ Department of Mathematics, Virginia Tech, Blacksburg, VA 24061-0123 (nloehr@vt.edu). 
operations on abaci correspond to algebraic manipulations of suitable determinants. One advantage of the combinatorial viewpoint is that it furnishes a convenient way to visualize, organize, and unify these determinant manipulations.

The rest of this paper is arranged as follows. Section 2 gives the necessary definitions concerning symmetric polynomials, antisymmetric polynomials, Schur polynomials, and abaci. Section 3 gives abacus proofs of the three Pieri rules. Section 4 uses abaci to prove that the combinatorial and algebraic definitions of Schur polynomials are equivalent. Section 5 proves two versions of the Littlewood-Richardson rule with the aid of abaci. Finally, section 6 derives Eğecioğlu and Remmel's combinatorial interpretation of the inverse Kostka matrix as well as a Pieri-like rule for the Schur coefficients in products of the form $s_{\nu} m_{\lambda}$, via abacus arguments.

2. Definitions. For a more detailed treatment of the concepts defined here, see $[10,12]$.

2.1. Symmetric and antisymmetric polynomials. Let $R=\mathbb{C}\left[x_{1}, \ldots, x_{N}\right]$ be the ring of polynomials in $N$ variables, where $N \geq 1$ is fixed throughout. The symmetric group $S_{N}$ acts on $R$ by permuting the variables. A polynomial $f \in R$ is called symmetric iff $\sigma \bullet f=f$ for all $\sigma \in S_{N} ; f$ is called antisymmetric iff $\sigma \bullet f=$ $\operatorname{sgn}(\sigma) f$ for all $\sigma \in S_{N}$.

The $k$ th power-sum symmetric polynomial is

$$
p_{k}\left(x_{1}, \ldots, x_{N}\right)=x_{1}^{k}+x_{2}^{k}+\cdots+x_{N}^{k} .
$$

The $k$ th elementary symmetric polynomial is

$$
e_{k}\left(x_{1}, \ldots, x_{N}\right)=\sum_{1 \leq i_{1}<i_{2}<\cdots<i_{k} \leq N} x_{i_{1}} x_{i_{2}} \cdots x_{i_{k}}=\sum_{\substack{k \text {-element subsets } \\ S \text { of }\{1, \ldots, N\}}}\left(\prod_{j \in S} x_{j}\right) .
$$

The $k$ th homogeneous symmetric polynomial is

$$
h_{k}\left(x_{1}, \ldots, x_{N}\right)=\sum_{1 \leq i_{1} \leq i_{2} \leq \cdots \leq i_{k} \leq N} x_{i_{1}} x_{i_{2}} \cdots x_{i_{k}}=\sum_{\substack{k \text {-element multisets } \\ M \text { of }\{1, \ldots, N\}}}\left(\prod_{j \in M} x_{j}^{n_{j}(M)}\right),
$$

where $n_{j}(M)$ denotes the number of times $j$ occurs in the multiset $M$. Let $\operatorname{Par}_{N}$ be the set of integer partitions $\lambda=\left(\lambda_{1} \geq \lambda_{2} \geq \cdots \geq \lambda_{N} \geq 0\right)$ with at most $N$ parts. For $\lambda \in$ $\operatorname{Par}_{N}$, the monomial symmetric polynomial indexed by $\lambda$, denoted $m_{\lambda}\left(x_{1}, \ldots, x_{N}\right)$, is the sum of all distinct monomials $x_{1}^{b_{1}} \cdots x_{N}^{b_{N}}$ whose exponent sequence $\left(b_{1}, \ldots, b_{N}\right)$ is a rearrangement of the parts of $\lambda$. The polynomials $m_{\lambda}$, as $\lambda$ ranges over $\operatorname{Par}_{N}$, form a basis for the vector space of symmetric polynomials in $R$.

If $\beta=\left(\beta_{1}, \ldots, \beta_{N}\right)$ is a list of $N$ distinct nonnegative integers, the monomial antisymmetric polynomial indexed by $\beta$ is

$$
a_{\beta}\left(x_{1}, \ldots, x_{N}\right)=\sum_{\sigma \in S_{N}} \operatorname{sgn}(\sigma) \prod_{k=1}^{N} x_{\sigma(k)}^{\beta_{k}}=\operatorname{det}\left\|x_{j}^{\beta_{i}}\right\|_{1 \leq i, j \leq N} .
$$

The polynomials $a_{\mu}$, where $\mu$ ranges over partitions in $\operatorname{Par}_{N}$ with distinct parts, form a basis for the vector space of antisymmetric polynomials in $R$. Each such $\mu$ can be written uniquely in the form $\mu=\lambda+\delta(N)$, where $\lambda \in \operatorname{Par}_{N}$ and $\delta(N)=$ $(N-1, N-2, \ldots, 2,1,0)$. We remark that $a_{\delta(N)}=\operatorname{det}\left\|x_{j}^{N-i}\right\|=\prod_{1 \leq r<s \leq N}\left(x_{r}-x_{s}\right)$ is the well-known Vandermonde determinant. 
2.2. Schur polynomials. Given $\lambda, \nu \in \operatorname{Par}_{N}$, we write $\nu \subseteq \lambda$ iff $\nu_{i} \leq \lambda_{i}$ for $1 \leq i \leq N$. Given $\nu \subseteq \lambda$, the skew shape $\lambda / \nu$ is the set of cells

$$
\lambda / \nu=\left\{(i, j) \in \mathbb{N}^{2}: 1 \leq i \leq N, \nu_{i}<j \leq \lambda_{i}\right\} .
$$

Given a total ordering $\preceq$ on $[N]=\{1,2, \ldots, N\}$, a semistandard tableau of shape $\lambda / \nu$ relative to $\preceq$ is a function $T: \lambda / \nu \rightarrow[N]$ such that $T(i, j) \preceq T(i, j+1)$ whenever $(i, j)$ and $(i, j+1)$ both lie in $\lambda / \nu$, and $T(i, j) \prec T(i+1, j)$ whenever $(i, j)$ and $(i+1, j)$ both lie in $\lambda / \nu$. Let $\operatorname{SSYT}_{\preceq}(\lambda / \nu)$ be the set of all such tableaux. The content monomial of $T$ is $x^{T}=\prod_{(i, j) \in \lambda / \nu} x_{T(i, j)}$. The content partition of $T$ is the unique partition obtained by rearranging the exponent vector of $x^{T}$.

The combinatorial definition of the skew Schur polynomial indexed by $\lambda / \nu$ is

$$
s_{\lambda / \nu}\left(x_{1}, \ldots, x_{N}\right)=\sum_{T \in \operatorname{SSYT}_{\preceq}(\lambda / \nu)} x^{T},
$$

where $\preceq$ is any total ordering of $[N]$. We will see later that the right side of (2.1) is indeed symmetric, which implies that the definition does not depend on the choice of the total ordering $\preceq$. Typically one takes the usual total ordering $1<2<\cdots<N$, but we will need other orderings for some of our abacus arguments.

Example 1. We can create a picture of a tableau $T \in \operatorname{SSYT}_{\preceq}(\lambda / \nu)$ by drawing a square containing $T(i, j)$ in row $i$, column $j$, for all $(i, j) \in \lambda / \nu$. Our convention is that the top row is row 1 . For example, here is a tableau of shape $(4,4,2) /(2)$ relative to the usual total ordering on [4]:

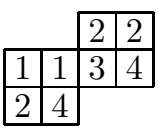

The algebraic definition of the Schur polynomial indexed by $\lambda \in \operatorname{Par}_{N}$ is

$$
s_{\lambda}\left(x_{1}, \ldots, x_{N}\right)=\frac{a_{\lambda+\delta(N)}\left(x_{1}, \ldots, x_{N}\right)}{a_{\delta(N)}\left(x_{1}, \ldots, x_{N}\right)}=\frac{\operatorname{det}\left\|x_{i}^{\lambda_{j}+N-j}\right\|_{i, j \in[N]}}{\operatorname{det}\left\|x_{i}^{N-j}\right\|_{i, j \in[N]}} .
$$

Later we show that this polynomial (which is evidently symmetric) is the same as the polynomial $s_{\lambda / 0}$ defined above.

2.3. Labeled abaci. A labeled abacus with $N$ beads is a word $w=\left(w_{k}: k \geq 0\right)$ such that the subword of nonzero letters in $w$ is a permutation of $1,2, \ldots, N$. The sign of $w$ is the sign of this permutation, the support of $w$ is the set $\operatorname{supp}(w)=\left\{k: w_{k} \neq 0\right\}$, and the weight of $w$ is $\operatorname{wt}(w)=\prod_{k \in \operatorname{supp}(w)} x_{w_{k}}^{k}$. Intuitively, $w_{k}=0$ means that position $k$ on the abacus is empty, whereas $w_{k}=i>0$ means that position $k$ on the abacus is occupied by a bead labeled $i$. Such a bead contributes a factor $x_{i}^{k}$ to the weight of the abacus. The sign of the abacus is the parity of the number of "bead inversions," which are pairs $j<k$ such that $w_{j}>w_{k}>0$. A labeled abacus $w$ determines a total ordering $\leq_{w}$ of the set $[N]$ defined by setting $i<_{w} j$ iff bead $i$ occurs to the right of bead $j$ on the abacus.

The shape of an abacus $w$, denoted $\operatorname{sh}(w)$, is the unique partition $\lambda \in \operatorname{Par}_{N}$ $\operatorname{such}$ that $\operatorname{supp}(w)$ is the set of parts of the partition $\lambda+\delta(N)$. We write $\operatorname{Abc}(\lambda)$ for the set of labeled abaci of shape $\lambda$. The $\operatorname{set} \operatorname{supp}(w)$ encodes the frontier of the diagram of $\operatorname{sh}(w)$ as follows. Start at the lower-left corner of an $N$-row diagram. 
For each $k=0,1,2, \ldots$, draw an east step if $k \notin \operatorname{supp}(w)$ and draw a north step if $k \in \operatorname{supp}(w)$. The shape to the left of this path is easily seen to be the diagram of $\operatorname{sh}(w)$. It is clear from the definitions that

$$
\sum_{w \in \operatorname{Abc}(\lambda)} \operatorname{sgn}(w) \operatorname{wt}(w)=\operatorname{det}\left\|x_{i}^{\lambda_{j}+N-j}\right\|_{i, j \in[N]}=a_{\lambda+\delta(N)}\left(x_{1}, \ldots, x_{N}\right) .
$$

In particular, $a_{\delta(N)}$ is the generating function for left-justified abaci in which the beads occupy positions $0,1, \ldots, N-1$. One can pass from an abacus $w \in \operatorname{Abc}(\lambda)$ to a justified abacus $J(w) \in \operatorname{Abc}(0)$ by moving the beads left by amounts given by the parts of $\lambda$.

Example 2. The word $w=510032046000 \cdots$ represents the following labeled abacus with 6 beads:

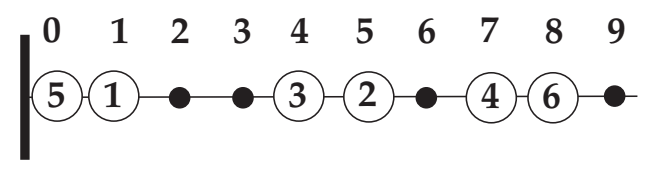

Here $N=6, \operatorname{sgn}(w)=-1, \operatorname{wt}(w)=x_{5}^{0} x_{1}^{1} x_{3}^{4} x_{2}^{5} x_{4}^{7} x_{6}^{8}, \operatorname{supp}(w)=\{0,1,4,5,7,8\}$, and $\operatorname{sh}(w)=(3,3,2,2,0,0)$. The total ordering of [6] determined by $w$ is

$$
6<_{w} 4<_{w} 2<_{w} 3<_{w} 1<_{w} 5 .
$$

3. Pieri rules. The Pieri rules give combinatorial prescriptions for the Schur expansions of products of the form $s_{\lambda} h_{k}, s_{\lambda} e_{k}$, and $s_{\lambda} p_{k}$. This section uses abaci to prove the antisymmetric versions of these Pieri rules. The usual Pieri rules for Schur polynomials follow by dividing our identities by $a_{\delta(N)}$ and using $(2.2)$.

3.1. Rule for multiplying by $\boldsymbol{e}_{\boldsymbol{k}}$. A skew shape $\mu / \lambda$ is a vertical $k$-strip iff $|\mu / \lambda|=k$ and for all $i, j_{1}, j_{2},\left(i, j_{1}\right) \in \mu / \lambda$ and $\left(i, j_{2}\right) \in \mu / \lambda$ imply $j_{1}=j_{2}$. Pictorially, this means that $\mu / \lambda$ consists of $k$ boxes, with no two in the same row.

Theorem 3.1 (Pieri rule for $e_{k}$ ). For all $\lambda \in \operatorname{Par}_{N}$ and all $k \geq 1$, $(3.1)$

$$
a_{\lambda+\delta(N)}\left(x_{1}, \ldots, x_{N}\right) e_{k}\left(x_{1}, \ldots, x_{N}\right)=\sum_{\substack{\mu \in \operatorname{Par}_{N}: \\ \text { is } \\ \text { a vertical } k \text {-strip }}} a_{\mu+\delta(N)}\left(x_{1}, \ldots, x_{N}\right) .
$$

Proof. The left side is the generating function for the signed, weighted set $X=$ $\operatorname{Abc}(\lambda) \times S_{k}([N])$, where $S_{k}([N])$ is the set of $k$-element subsets of $[N]$, and for $(w, S) \in X, \operatorname{sgn}(w, S)=\operatorname{sgn}(w)$ and $\operatorname{wt}(w, S)=\operatorname{wt}(w) \prod_{j \in S} x_{j}$. We define a signreversing, weight-preserving involution $I$ on $X$ whose fixed points correspond to the right side of (3.1).

Given $(w, S) \in X$, scan the abacus $w$ from right to left. Each time a bead in $S$ is encountered in the scan, move the bead one step to the right. Two cases are possible: either a bead collides with another bead during this scan, or no bead collision occurs. The first case occurs iff there exist $j \in S$ and $k \notin S$ with bead $k$ lying one step to the right of bead $j$ on $w$. Define $I(w, S)=\left(w^{\prime}, S^{\prime}\right)$, where $w^{\prime}$ is $w$ with beads $j$ and $k$ interchanged and $S^{\prime}=(S-\{j\}) \cup\{k\}$; here, $j$ and $k$ are the beads involved in the first bead collision. It is immediately verified that $\operatorname{sgn}\left(w^{\prime}, S^{\prime}\right)=-\operatorname{sgn}(w, S)$, $\operatorname{wt}\left(w^{\prime}, S^{\prime}\right)=\operatorname{wt}(w, S)$, and $I\left(w^{\prime}, S^{\prime}\right)=(w, S)$.

Now consider the case where no bead collision occurs; we set $I(w, S)=(w, S)$ in this case. The bead motions transform $w$ into a new abacus $w^{*}$ such that $\operatorname{wt}\left(w^{*}\right)=$ 
$\operatorname{wt}(w, S)$ and $\operatorname{sgn}\left(w^{*}\right)=\operatorname{sgn}(w, S)$. Write $\mu=\operatorname{sh}\left(w^{*}\right)$. Recall that the parts of $\lambda$ (resp., $\mu$ ) give the amounts by which beads must be moved along $w$ (resp., $w^{*}$ ) to reach a justified abacus. It follows that $\mu$ is obtained from $\lambda$ by adding 1 to $k$ distinct parts of $\lambda$. This means that the skew shape $\mu / \lambda$ is a vertical $k$-strip. Conversely, any abacus $w^{*}$ counted by the right side of (3.1) arises from a unique fixed point $(w, S) \in X$ via the indicated bead motions. So, the bead motion rule defines a signpreserving, weight-preserving bijection from the fixed points of $I$ to the set of objects counted by the right side of (3.1).

Example 3. Let $N=6, \lambda=(3,3,2,2,0,0), k=3$, and

$$
(w, S)=(51003204600 \cdots,\{1,2,3\}) .
$$

Moving beads 2, 3, and 1 (in this order) leads to a new abacus $w^{*}=(50100324600 \cdots)$ in $\operatorname{Abc}(\mu)$, where $\mu=(3,3,3,3,1,0)$ and $\mu / \lambda$ is a vertical 3 -strip. Note that $\operatorname{sgn}\left(w^{*}\right)=$ $\operatorname{sgn}(w)$ and $\operatorname{wt}\left(w^{*}\right)=\operatorname{wt}(w) x_{1} x_{2} x_{3}=\operatorname{wt}(w, S)$.

On the other hand, if $S=\{3,5,6\}$ instead, then bead 3 collides with bead 2 . Therefore,

$$
I(w, S)=\left(w^{\prime}, S^{\prime}\right)=(51002304600 \cdots,\{2,5,6\}) .
$$

Note that $\operatorname{sgn}\left(w^{\prime}\right)=-\operatorname{sgn}(w)$ and $\operatorname{wt}(w, S)=\operatorname{wt}(w) x_{3} x_{5} x_{6}=\operatorname{wt}\left(w^{\prime}\right) x_{2} x_{5} x_{6}=$ $\operatorname{wt}\left(w^{\prime}, S^{\prime}\right)$.

3.2. Rule for multiplying by $\boldsymbol{h}_{\boldsymbol{k}}$. A skew shape $\mu / \lambda$ is a horizontal $k$-strip iff $|\mu / \lambda|=k$ and for all $i_{1}, i_{2}, j,\left(i_{1}, j\right) \in \mu / \lambda$ and $\left(i_{2}, j\right) \in \mu / \lambda$ imply $i_{1}=i_{2}$. Pictorially, this means that $\mu / \lambda$ consists of $k$ boxes, with no two in the same column.

Theorem 3.2 (Pieri rule for $h_{k}$ ). For all $\lambda \in \operatorname{Par}_{N}$ and all $k \geq 1$,

$$
a_{\lambda+\delta(N)}\left(x_{1}, \ldots, x_{N}\right) h_{k}\left(x_{1}, \ldots, x_{N}\right)=\sum_{\substack{\mu \in \operatorname{Par}_{N}: \\ \text { is } \\ \text { a horizontal k-strip }}} a_{\mu+\delta(N)}\left(x_{1}, \ldots, x_{N}\right) .
$$

Proof. The left side counts signed, weighted objects in the set $X=\operatorname{Abc}(\lambda) \times$ $M_{k}([N])$, where $M_{k}([N])$ is the set of $k$-element multisets using letters in [N], and for $(w, M) \in X, \operatorname{sgn}(w, M)=\operatorname{sgn}(w)$ and $\operatorname{wt}(w, M)=\operatorname{wt}(w) \prod_{j \in M} x_{j}^{n_{j}(M)}$. As before, we define an involution $I$ on $X$ whose fixed points correspond to the right side of (3.2).

Given $(w, M) \in X$, scan the abacus $w$ from left to right. When a bead $j \in M$ is encountered in the scan, move the bead $n_{j}(M)$ steps to the right, one step at a time. If all bead motions are completed with no bead collisions, $(w, M)$ is a fixed point of $I$. The bead motions on $w$ produce an abacus $w^{*}$ with $\operatorname{sgn}\left(w^{*}\right)=\operatorname{sgn}(w)$ and $\operatorname{wt}(w, M)=\operatorname{wt}\left(w^{*}\right)$. Setting $\mu=\operatorname{sh}\left(w^{*}\right)$, it follows easily from the bead motion rule that $\mu / \lambda$ is a horizontal $k$-strip. Conversely, any abacus of such a shape arises from a unique fixed point $(w, M) \in X$.

On the other hand, suppose a bead collision does occur. Say the first collision happens when bead $j$ hits a bead $k$ that is located $p \leq n_{j}(M)$ positions to the right of bead $j$ 's initial position. Define $I(w, M)=\left(w^{\prime}, M^{\prime}\right)$, where $w^{\prime}$ is $w$ with beads $j$ and $k$ interchanged and $M^{\prime}$ is obtained from $M$ by setting $n_{j}\left(M^{\prime}\right)=n_{j}(M)-p$, $n_{k}\left(M^{\prime}\right)=n_{k}(M)+p$, and $n_{i}\left(M^{\prime}\right)=n_{i}(M)$ for all $i \neq j, k$. One sees that this definition preserves weights and reverses signs, and $I(I(w, M))=(w, M)$.

Example 4. Let $N=6, \lambda=(3,3,2,2,0,0), k=3$, and

$$
(w, M)=(51003204600 \cdots,[1,1,2]) .
$$


Moving bead 1, then bead 1 , then bead 2 gives a new abacus $w^{*}=(50013024600 \cdots)$ in $\operatorname{Abc}(\mu)$, where $\mu=(3,3,3,2,2,0)$ and $\mu / \lambda$ is a horizontal 3 -strip. Note that $\operatorname{sgn}\left(w^{*}\right)=\operatorname{sgn}(w)$ and $\operatorname{wt}\left(w^{*}\right)=\operatorname{wt}(w) x_{1} x_{1} x_{2}=\operatorname{wt}(w, M)$.

On the other hand, if $M=[1,2,3]$ instead, then bead 3 collides with bead 2 since bead 3 moves first. Therefore,

$$
I(w, M)=\left(w^{\prime}, M^{\prime}\right)=(51002304600 \cdots,[1,2,2]) .
$$

Note that $\operatorname{sgn}\left(w^{\prime}\right)=-\operatorname{sgn}(w)$ and $\operatorname{wt}(w, M)=\operatorname{wt}(w) x_{1} x_{2} x_{3}=\operatorname{wt}\left(w^{\prime}\right) x_{1} x_{2} x_{2}=$ $\operatorname{wt}\left(w^{\prime}, M^{\prime}\right)$.

3.3. Rule for multiplying by $\boldsymbol{p}_{\boldsymbol{k}}$. A ribbon is a skew shape that can be formed by starting at a given square, repeatedly moving left or down one step at a time, and including all squares visited in this way. A $k$-ribbon is a ribbon of size $k$. If a ribbon $R$ occupies $r$ distinct rows, we set $\operatorname{sgn}(R)=(-1)^{r-1}$.

TheORem 3.3 (Pieri rule for $p_{k}$ ). For all $\lambda \in \operatorname{Par}_{N}$ and all $k \geq 1$,

$$
a_{\lambda+\delta(N)}\left(x_{1}, \ldots, x_{N}\right) p_{k}\left(x_{1}, \ldots, x_{N}\right)=\sum_{\substack{\mu \in \operatorname{Par}_{N}: \\ \mu / \lambda \text { is a k-ribbon }}} \operatorname{sgn}(\mu / \lambda) a_{\mu+\delta(N)}\left(x_{1}, \ldots, x_{N}\right) .
$$

Proof. The left side counts signed, weighted objects in the set $X=\operatorname{Abc}(\lambda) \times[N]$, where we set $\operatorname{sgn}(w, j)=\operatorname{sgn}(w)$ and $\operatorname{wt}(w, j)=\operatorname{wt}(w) x_{j}^{k}$ for $(w, j) \in X$. We define an involution $I$ on $X$ as follows. Given $(w, j) \in X$, let $r$ be the unique position with $w_{r}=j$.

First consider the case where $w_{r+k}=0$. In this case, let $I(w, j)=(w, j)$, and define $w^{*}$ by setting $w_{r}^{*}=0, w_{r+k}^{*}=j$, and $w_{i}^{*}=w_{i}$ for all $i \neq r, r+k$. Intuitively, to go from $(w, j)$ to $w^{*}$, bead $j$ "jumps" $k$ positions to the right in a single move. Thus $\operatorname{supp}\left(w^{*}\right)=(\operatorname{supp}(w)-\{r\}) \cup\{r+k\}$. Let $\mu=\operatorname{sh}\left(w^{*}\right)$. Using the relationship between $\operatorname{supp}(w)\left(\right.$ resp., $\left.\operatorname{supp}\left(w^{*}\right)\right)$ and the frontier of $\lambda$ (resp., $\mu$ ), described in section 2.3, it is easy to see that $\mu / \lambda$ must be a $k$-ribbon. Furthermore, $\operatorname{sgn}\left(w^{*}\right)=\operatorname{sgn}(\mu / \lambda) \operatorname{sgn}(w)$ and $\operatorname{wt}\left(w^{*}\right)=\operatorname{wt}(w) x_{j}^{k}=\mathrm{wt}(w, j)$. Finally, every abacus of shape $\mu$ (where $\mu / \lambda$ is any $k$-ribbon) arises in this way from a unique fixed point $(w, j) \in X$. Thus, the fixed points of $I$ are enumerated by the right side of (3.3).

Now consider the case where $w_{r+k}=i>0$ so that the bead-jumping move causes bead $j$ to collide with bead $i$. Set $I(w, j)=\left(w^{\prime}, i\right)$, where $w^{\prime}$ is $w$ with beads $i$ and $j$ switched. The permutations associated to $w$ and to $w^{\prime}$ differ by the transposition $(i, j), \operatorname{so} \operatorname{sgn}\left(w^{\prime}\right)=-\operatorname{sgn}(w)$. Furthermore,

$$
\mathrm{wt}(w, j)=\left(x_{j}^{r} x_{i}^{r+k} \cdots\right) x_{j}^{k}=\left(x_{i}^{r} x_{j}^{r+k} \cdots\right) x_{i}^{k}=\operatorname{wt}(I(w, j)) .
$$

Finally, it is clear that $I\left(w^{\prime}, i\right)=(w, j)$, so $I$ is an involution.

Example 5. Suppose $N=6, \lambda=(3,3,2,2,0,0), k=3$, and $w=51003204600 \cdots$. Let us compute $I(w, j)$ for $1 \leq j \leq 6$. For $j=1$, bead 1 jumps 3 positions to the right and collides with bead 3 . So

$$
I(w, 1)=(53001204600 \cdots, 3) .
$$

Similarly, $I(w, 2)=(51003604200 \cdots, 6)$ and $I(w, 3)=(51004203600 \cdots, 4)$.

On the other hand, when $j=4$, bead 4 jumps 3 positions to position 10, which is empty. So

$$
I(w, 4)=(w, 4), \quad w^{*}=5100320060400 \cdots
$$


Similarly, $(w, 5)$ and $(w, 6)$ are fixed points of $I$ with associated abaci

$$
w^{*}=01053204600 \cdots \text { and } w^{*}=51003204000600 \cdots .
$$

The following partition diagrams show that the shapes of each of these new abaci are obtained from $\lambda$ by adding a 3-ribbon:
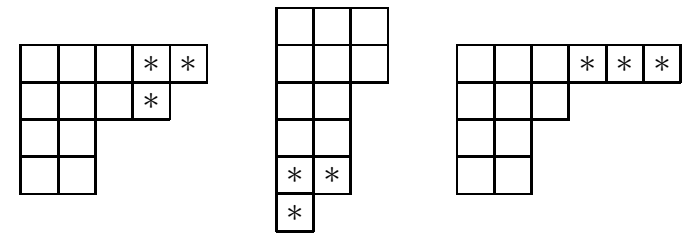

Observe that the shape $\mu=(3,3,2,2,1,1,1)$ does not appear, even though $\mu / \lambda$ is a 3-ribbon, because $\mu \notin \operatorname{Par}_{6}$.

Remark. The "bead-jumping" motion in the previous proof can also be visualized by drawing the abacus $w=w_{0} w_{1} w_{2} \cdots$ using $k$ separate runners. For $0 \leq r<k$, the $r$ th runner of $w$ is the subword $w_{r} w_{k+r} w_{2 k+r} w_{3 k+r} \cdots$. For example, the 3-runner version of the abacus $w=51003204600 \cdots$ looks like this:

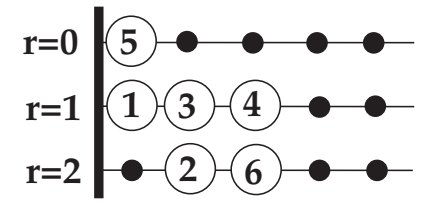

In this formulation, we need only move bead $j$ one step along its runner. If this causes a collision with bead $i$, we switch $i$ and $j$ to obtain the matched object; otherwise we get a fixed point.

4. Equivalence of two definitions of Schur polynomials. This section uses abaci to prove that the combinatorial definition (2.1) (with $\nu=0$ ) agrees with the algebraic definition (2.2) of Schur polynomials. For a different combinatorial proof and a discussion of other proofs of this result, see [4]. We begin by describing an operation on tableaux that proves that the right side of (2.1) does not depend on the total ordering $\preceq$. Variations of this operation will play a central role in the abacus proofs appearing below.

4.1. Symmetry of skew Schur polynomials. We recall a result whose (wellknown) proof uses the combinatorial "crystal operations" of Kashiwara [8, 9]. An alternate combinatorial proof was given by Bender and Knuth [2].

TheOREM 4.1. For all $N \geq 1$ and all skew shapes $\lambda / \nu$, the polynomial

$$
\sum_{T \in \operatorname{SSYT}_{\preceq}(\lambda / \nu)} x^{T}
$$

does not depend on the total ordering $\preceq$ of $[N]$.

Proof. Suppose $\leq_{1}$ is a total ordering of $[N]$ and $i, j$ are adjacent elements in this ordering with $i>_{1} j$. Let $\leq_{2}$ be the total ordering that agrees with $\leq_{1}$ except that $i<_{2}$ $j$. We will define a weight-preserving bijection $\phi: \operatorname{SSYT}_{\leq_{1}}(\lambda / \nu) \rightarrow \operatorname{SSYT}_{\leq_{2}}(\lambda / \nu)$. This will prove that

$$
\sum_{T \in \mathrm{SSYT}_{\leq_{1}}(\lambda / \nu)} x^{T}=\sum_{U \in \mathrm{SSYT}_{\leq_{2}}(\lambda / \nu)} x^{U}
$$


Any total ordering of $[N]$ can be transformed into any other total ordering by a sequence of moves of this type, so the theorem will follow once $\phi$ is constructed.

Define the word of a tableau $T$, denoted $w(T)$, to be the concatenation of the rows of $T$ from bottom to top. For instance, the tableau in Example 1 has word 24113422. Note $T$ is recoverable from $w(T)$ if we know the shape $\lambda / \nu$ and the ordering $\preceq$. Now suppose $T \in \operatorname{SSYT}_{\leq_{1}}(\lambda / \nu)$ has word $w$. We create a new word $w^{\prime}$ by modifying the subword of $i$ 's and $j$ 's in $w$. First, replace each $i$ by a left parenthesis and each $j$ by a right parenthesis. Match left and right parentheses in the resulting string of parentheses, and ignore these matched pairs of parentheses in what follows. The unmatched parentheses must consist of $a \geq 0$ right parentheses followed by $b \geq 0$ left parentheses. Change this configuration to a string of $b$ right parentheses followed by $a$ left parentheses. Finally, construct $w^{\prime}$ by replacing all left parentheses (matched and unmatched) by $j$ 's and all right parentheses by $i$ 's. It is routine to check that the word $w^{\prime}$ always is the word of a tableau $T^{\prime} \in \operatorname{SSYT}_{\leq_{2}}(\lambda / \nu)$ and $x^{T^{\prime}}=x^{T}$.

Example 6. Let $T$ be the tableau in Example 1, and let $<_{1}$ be the standard total order on [4]. Take $i=2$ and $j=1$, so $<_{2}$ is the total order $2<_{2} 1<_{2} 3<_{2} 4$. The subword of 2's and 1's in $w=w(T)=24113422$ turns into the parenthesis string ()$)$ ( ( . Switching the frequencies of unmatched parentheses produces the string ()$))\left(\right.$, so $w^{\prime}=14223421$ and $T^{\prime}$ is

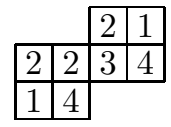

Note that $T^{\prime} \in \operatorname{SSYT}_{\leq_{2}}((4,4,2) /(2))$ and $x^{T^{\prime}}=x^{T}$.

One easily deduces from this theorem that the polynomial in (2.1) is a symmetric polynomial.

4.2. Tableaux versus abaci.

Theorem 4.2. For all $\lambda \in \operatorname{Par}_{N}$,

$$
a_{\delta(N)}\left(x_{1}, \ldots, x_{N}\right) s_{\lambda / 0}\left(x_{1}, \ldots, x_{N}\right)=a_{\lambda+\delta(N)}\left(x_{1}, \ldots, x_{N}\right) .
$$

So $s_{\lambda / 0}\left(x_{1}, \ldots, x_{N}\right)=s_{\lambda}\left(x_{1}, \ldots, x_{N}\right)$.

Proof. Let $X$ be the set of pairs $(v, T)$, where $v \in \operatorname{Abc}(0)$ and $T \in \operatorname{SSYT}_{\leq_{v}}(\lambda)$. Define $\operatorname{sgn}(v, T)=\operatorname{sgn}(v)$ and $\operatorname{wt}(v, T)=\operatorname{wt}(v) x^{T}$. Using the previous theorem, we see that the left side of (4.1) enumerates the signed, weighted set $X$. It suffices to define an involution $I$ on $X$ whose fixed points correspond to the weighted set $\operatorname{Abc}(\lambda)$.

Given $(v, T) \in X, T$ determines a sequence of bead motions on $v$ by reading $w(T)$ from right to left and moving bead $k$ one step right each time the symbol $k$ is seen. If these bead motions cause a collision, define $I(v, T)=\left(v^{\prime}, T^{\prime}\right)$ as follows. Say the first collision occurs when bead $i$ bumps into bead $j$ (so $i>{ }_{v} j$ must be adjacent beads in $v)$. Let $v^{\prime}$ be $v$ with beads $i$ and $j$ switched, so $\operatorname{sgn}\left(v^{\prime}\right)=-\operatorname{sgn}(v)$ and $\operatorname{wt}\left(v^{\prime}\right) x_{j}=$ wt $(v) x_{i}$. We transform $T$ into $T^{\prime}$ using the following variant of the parenthesismatching operation. As before, replace each $i$ in $w(T)$ by a left parenthesis, each $j$ by a right parenthesis, and ignore all other symbols. The unmatched parentheses in the resulting string must consist of $a \geq 0$ right parentheses followed by $b \geq 0$ left parentheses. It is easy to see that $b>0$ since otherwise bead $i$ would never bump into bead $j$. Indeed, the first bead collision occurs when we reach the rightmost unmatched left parenthesis (occurrence of $i$ ) in the word of $T$. Now change the subword of unmatched parentheses from " $)^{a}(b " \text { to " })^{b-1}\left({ }^{a+1} "\right.$ and then convert all 
left parentheses to $j$ 's and all right parentheses to $i$ 's. As before, one sees that the resulting word $i s$ the word of a tableau $T^{\prime} \in \operatorname{SSYT}_{\leq_{v^{\prime}}}(\lambda)$. Now $T^{\prime}$ has one less $i$ than $T$ and one more $j$ than $T$. It follows that $\operatorname{wt}\left(v^{\prime}, T^{\prime}\right) \stackrel{v^{\prime}}{=} \operatorname{wt}(v, T)$. Furthermore, consider what happens when we calculate $I\left(v^{\prime}, T^{\prime}\right)$. Bead $j$ on $v^{\prime}$ moves the same way as bead $i$ did on $v$ (and vice versa) until we reach the rightmost unmatched parenthesis (relative to $i$ and $j$ ) in $w\left(T^{\prime}\right)$. When this symbol is reached, bead $j$ bumps into bead $i$ on $v^{\prime}$, just as bead $i$ bumped into bead $j$ on $v$. To compute $I\left(v^{\prime}, T^{\prime}\right)$, we will therefore apply the parenthesis modification rule to the $i$ 's and $j$ 's in $w\left(T^{\prime}\right)$. This will restore the word of $T$, so $I\left(v^{\prime}, T^{\prime}\right)=(v, T)$ as desired.

To finish the proof, let us analyze the fixed points of $I$, which are (by definition) the objects $(v, T)$ where no bead collisions occur. Let $i$ be the last entry in the top row of $T$, which is the rightmost letter in $w(T)$. We must first move bead $i$ one step right on $v$. This move will already cause a collision (since $v$ is justified) unless $i=v_{N-1}$. Since $v_{N-1}$ is the smallest letter relative to $<_{v}$ and $T$ is semistandard, $i=v_{N-1}$ iff all entries in the top row of $T$ are equal to $v_{N-1}$. In this situation, we will move the rightmost bead $v_{N-1}$ to the right $\lambda_{1}$ positions with no collisions.

Now we repeat the argument on the second row of $T$. The rightmost entry $j$ in this row cannot be $v_{N-1}$ (otherwise the columns of $T$ would not strictly increase reading down). The only way to avoid an immediate bead collision is when $j=v_{N-2}$, in which case all entries in the second row of $T$ must equal $v_{N-2}$. In this situation, bead $v_{N-2}$ will move right $\lambda_{2}$ positions with no collisions.

Continuing similarly, we see that $(v, T)$ will have no collisions iff for all $k$, the $k$ th row of $T$ consists of $\lambda_{k}$ copies of the $k$ th smallest letter $v_{N-k}$ relative to $<_{v}$. Moving the beads on $v$ according to $T$ has the effect of unjustifying $v$ to an abacus $v^{*} \in \operatorname{Abc}(\lambda)$. The association $(v, T) \mapsto v^{*}$ is evidently a weight-preserving, signpreserving bijection between the fixed points of $I$ and $\operatorname{Abc}(\lambda)$. Thus the proof is complete.

Example 7. For $N=7$ and $\lambda=(7,7,5)$, we have

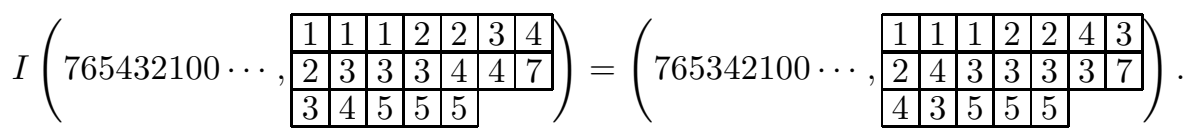

In this example, bead $i=4$ immediately bumps into bead $j=3$, so we modify the subword of 3's and 4's in $w(T)$ as follows:

$$
343334434 \rightarrow \quad)()))(()(\rightarrow \quad)()((()(\rightarrow \quad 434333343
$$

Now suppose $T$ is the "superstandard" tableau of shape $\lambda$ with word

$$
333332222221111111 .
$$

Then $(765432100 \cdots, T)$ is a fixed point of $I$ with associated abacus

$$
v^{*}=7654000003002100 \cdots \in \operatorname{Abc}(\lambda) .
$$

5. Littlewood-Richardson rules. This section gives abacus-based proofs of two versions of the celebrated Littlewood-Richardson rules. For information on other proofs, which often utilize connections between tableaux and the plactic monoid, see [6] and the notes to Appendix 1 of Chapter 7 of [12]. 
5.1. Schur expansion of skew Schur polynomials. In the last section, we used tableaux of partition shape to encode bead motions on an initially justified abacus. By generalizing this procedure to tableaux of skew shape, we are led naturally to an abacus proof of the Littlewood-Richardson rule. To state the rule, we need one more definition. Given a total ordering $x_{1} \prec x_{2} \prec \cdots \prec x_{N}$ on $[N]$, we say that a word $w=w_{1} w_{2} \cdots w_{m}$ has partition content relative to $\preceq$ iff for all $i<N$, the number of $x_{i}$ 's in $w$ is at least as great as the number of $x_{i+1}$ 's in $w$.

TheOREM 5.1. For all $\lambda, \mu, \nu \in \operatorname{Par}_{N}$, let $c_{\nu, \mu}^{\lambda}$ be the number of tableaux $T \in$ $\mathrm{SSYT}_{\leq}(\lambda / \nu)$ with content $\mu$ such that every suffix of $w(T)$ has partition content relative to $\leq$ (where $\leq$ is the usual numerical ordering on $[N]$ ). Then

$$
s_{\lambda / \nu}\left(x_{1}, \ldots, x_{N}\right)=\sum_{\mu \in \operatorname{Par}_{N}} c_{\nu, \mu}^{\lambda} s_{\mu}\left(x_{1}, \ldots, x_{N}\right) .
$$

Proof. Multiplying the identity by $a_{\delta(N)}$, it suffices to prove

$$
a_{\delta(N)}\left(x_{1}, \ldots, x_{N}\right) s_{\lambda / \nu}\left(x_{1}, \ldots, x_{N}\right)=\sum_{\mu \in \operatorname{Par}_{N}} c_{\nu, \mu}^{\lambda} a_{\mu+\delta(N)}\left(x_{1}, \ldots, x_{N}\right) .
$$

By Theorem 4.1, we can model the left side of this identity by the set $X$ of objects $(v, T)$, where $v \in \operatorname{Abc}(0)$ and $T \in \operatorname{SSYT}_{\leq v}(\lambda / \nu)$.

Given $(v, T) \in X$, we define the involution $I(v, T)=\left(v^{\prime}, T^{\prime}\right)$ just as in the previous proof. Read the symbols in $w(T)$ from right to left and move the corresponding beads on the abacus $v$. If a bead collision first occurs when bead $i$ bumps into bead $j$ (where $i>_{v} j$ are adjacent beads on $v$ ), let $v^{\prime}$ be $v$ with beads $i$ and $j$ switched. Construct $w\left(T^{\prime}\right)$ from $w(T)$ by modifying the subword of unmatched parentheses (relative to $i$ and $j$ ) by the same algorithm used in the proof of Theorem 4.2. This modification lowers the number of $i$ 's in $T$ by 1 and increases the number of $j$ 's in $T$ by 1 . So $\operatorname{wt}\left(v^{\prime}, T^{\prime}\right)=\operatorname{wt}(v, T)$. One also sees that $\operatorname{sgn}\left(v^{\prime}, T^{\prime}\right)=-\operatorname{sgn}(v, T)$ and $I\left(v^{\prime}, T^{\prime}\right)=(v, T)$. So $I$ is an involution that cancels all objects in which bead collisions occur.

Let us now examine the objects $(v, T) \in X$ for which no collisions occur. Since $v$ is justified and the bead motions arise from a right-to-left scan of $w(T)$, one easily sees that there are no bead collisions iff every suffix of $T$ has partition content relative to $\leq_{v}$. Now moving the beads on $v$ according to $w(T)$ defines a bijection between such objects (the fixed points of $I$ ) and certain abaci $v^{*}$. We can obtain all the fixed points of $I$ from fixed points of the form $\left(v^{0}, T\right)$, where $v^{0}=(N, N-1, \ldots, 2,1,0,0, \ldots)$, $\leq_{v^{0}}$ is the usual ordering $\leq$ on $[N]$, and $T \in \operatorname{SSYT}_{\leq}(\lambda / \nu)$ is a tableau satisfying the conditions in the theorem statement. We need only permute the labels in $v^{0}$ by some permutation in $S_{N}$ and permute the entries of $T$ in the same way. If $T$ has content $\mu \in \operatorname{Par}_{N}$, the $N$ ! fixed points associated to $\left(v^{0}, T\right)$ in this way will map to $N$ ! abaci $v^{*} \in \operatorname{Abc}(\mu)$ that jointly contribute one copy of $a_{\mu+\delta(N)}\left(x_{1}, \ldots, x_{N}\right)$ to the generating function for the fixed points of $I$. The total number of times this term appears in the generating function is precisely the number of tableaux $T \in \mathrm{SSYT}_{\leq}(\lambda / \nu)$ of content $\mu$ satisfying the conditions in the theorem. Since the generating function for $X$ must equal the generating function for the fixed points of $I$, the proof is complete.

One nice feature of the abacus proof is that it gives an intuitive combinatorial explanation for the condition on suffixes of $w(T)$ that appears in the LittlewoodRichardson rule. If desired, the abacus proof can be translated into a purely algebraic proof involving determinants (see [11] for a proof along these lines). 
Example 8. Let us compute $I\left(v^{0}, T\right)$, where $v^{0}=5432100 \cdots, N=5$, and $T$ is

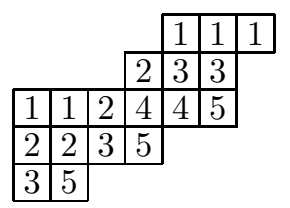

Moving beads on $v^{0}$ according to the word $w(T)=352235112445233111$, bead 3 bumps into bead 2 when we have scanned the suffix 3111 (which is the shortest suffix of $w(T)$ without partition content). We therefore modify the subword of 2's and 3's in $w(T)$ as follows:

$$
32232233 \rightarrow())())((\rightarrow \quad))()(((\rightarrow 23323222 .
$$

Thus $w\left(T^{\prime}\right)=253325113445222111$, and $I\left(v^{0}, T\right)=\left(v^{\prime}, T^{\prime}\right)$, where

$$
\begin{aligned}
& \begin{array}{|l|l|l|}
\hline 1 & 1 & 1 \\
\hline
\end{array}
\end{aligned}
$$

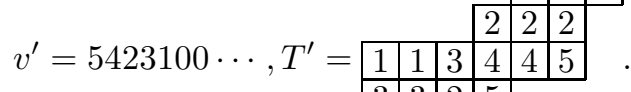

$$
\begin{aligned}
& \begin{array}{|l|l|l|l|}
\hline 3 & 3 & 2 & 5 \\
\hline 2 & 5 & &
\end{array}
\end{aligned}
$$

Observe that $\operatorname{wt}\left(v^{0}, T\right)=\operatorname{wt}\left(v^{\prime}, T^{\prime}\right)=x_{1}^{9} x_{2}^{7} x_{3}^{6} x_{4}^{3} x_{5}^{3}, \operatorname{sgn}\left(v^{\prime}, T^{\prime}\right)=-1=-\operatorname{sgn}\left(v^{0}, T\right)$, and $I\left(v^{\prime}, T^{\prime}\right)=\left(v^{0}, T\right)$.

5.2. Schur expansion of products of Schur polynomials. By a well-known argument using the Hall scalar product [10], one can show that

$$
s_{\nu}\left(x_{1}, \ldots, x_{N}\right) s_{\mu}\left(x_{1}, \ldots, x_{N}\right)=\sum_{\lambda \in \operatorname{Par}_{N}} c_{\nu, \mu}^{\lambda} s_{\lambda}\left(x_{1}, \ldots, x_{N}\right),
$$

where $c_{\nu, \mu}^{\lambda}$ are the Littlewood-Richardson coefficients defined in Theorem 5.1. If we try to prove this identity with abaci, we are led to an alternate combinatorial description of these coefficients.

TheOREM 5.2. For all $\lambda, \mu, \nu \in \operatorname{Par}_{N}$, the coefficient $c_{\nu, \mu}^{\lambda}=c_{\mu, \nu}^{\lambda}$ in (5.1) is the number of semistandard tableaux $T$ of shape $\mu$ and content $\lambda-\nu=\left(\lambda_{i}-\nu_{i}: 1 \leq i \leq N\right)$ such that $w(T)=w_{1} w_{2} \cdots w_{m}$ satisfies the following condition: for all $k \leq m$, the exponent vector of the monomial $\prod_{j=1}^{N} x_{j}^{\nu_{j}} \prod_{i=k}^{m} x_{w_{i}}$ is a partition.

Proof. This result follows routinely from Theorem 5.1 upon recognizing that $s_{\nu} s_{\mu}$ is the skew Schur polynomial indexed by a suitable shape $\alpha$ that consists of disjoint copies of $\nu$ and $\mu$. However, we can also prove (5.1) directly using abaci. We sketch this proof, which is quite similar to the proof of Theorem 5.1. We will prove that

$$
a_{\nu+\delta(N)} s_{\mu}=\sum_{\lambda} \tilde{c}_{\nu, \mu}^{\lambda} a_{\lambda+\delta(N)},
$$

where $\tilde{c}_{\nu, \mu}^{\lambda}$ is the combinatorial quantity defined in the statement of Theorem 5.2. Model the left side by the set $X$ of pairs $(v, T)$, where $v \in \operatorname{Abc}(\nu)$ and $T \in \operatorname{SSYT}_{\leq_{v}}(\mu)$. To define $I(v, T)=\left(v^{\prime}, T^{\prime}\right)$, scan the symbols in $w(T)$ from right to left and move the associated beads on $v$. Suppose a bead collision first occurs when bead $i$ bumps into bead $j$ (where $i>_{v} j$ ). Let $c \geq 1$ be the number of positions separating $i$ and $j$ in $v$. Let $v^{\prime}$ be $v$ with beads $i$ and $j$ interchanged. To find $T^{\prime}$, we use a variation of the 
parenthesis constructions used before. Here the subword of unmatched parentheses in $w(T)$ (relative to $i$ and $j$ ) must have the form ") ${ }^{a}(b$ " where $b \geq c$; the $c$ th unmatched left parenthesis from the right end is the one that causes the bead collision. We replace this subword by " $)^{b-c}\left({ }^{a+c "}\right.$ " and convert back as usual to get $w\left(T^{\prime}\right)$. One checks that this action preserves weights, reverses signs, and is involutory.

If no bead collision occurs, $(v, T)$ is a fixed point of $I$. As in the previous proof, all such fixed points arise from fixed points of the form $\left(v^{0}, T\right)$, where $J\left(v^{0}\right)=(N, N-$ $1, \ldots, 2,1,0, \ldots)$, by permuting the labels in $v^{0}$ and $T$. It is immediate that $\left(v^{0}, T\right)$ is a fixed point iff $w(T)$ satisfies the condition in the theorem statement. The abaci $v^{*}$ associated to all the fixed points produce various terms $a_{\nu+\beta+\delta(N)}$, where $\beta$ is the content of $T$. The number of times $a_{\lambda+\delta(N)}$ occurs in the generating function for the fixed points of $I$ is exactly the number of $T$ with content $\beta=\lambda-\nu$ satisfying the suffix condition in the theorem.

6. Inverse Kostka matrix. In section 3.3, we used abaci to find the Schur expansion of the symmetric polynomial $s_{\lambda} p_{k}=s_{\lambda} m_{(k)}$. We can generalize that calculation to find the Schur expansion of any monomial symmetric polynomial $m_{\lambda}$. This leads to an abacus-based derivation of Eğecioğlu and Remmel's combinatorial formula for the inverse Kostka matrix [5]. This formula involves special rim-hook tableaux, which we now define.

A rim-hook tableau of shape $\lambda / \nu$ and content $\alpha=\left(\alpha_{1}, \ldots, \alpha_{s}\right)$ is a sequence $S$ of partitions

$$
\nu=\mu^{0} \subseteq \mu^{1} \subseteq \mu^{2} \subseteq \cdots \subseteq \mu^{s}=\lambda
$$

such that $\mu^{i} / \mu^{i-1}$ is an $\alpha_{i}$-ribbon for $1 \leq i \leq s$. The sign of $S$ is $\operatorname{sgn}(S)=$ $\prod_{i=1}^{s} \operatorname{sgn}\left(\mu^{i} / \mu^{i-1}\right)$. The type of $S$ is the partition obtained by sorting the parts of $\alpha . S$ is called special iff every nonempty ribbon $\mu^{i} / \mu^{i-1}$ begins in the leftmost cell of some row of the skew shape $\lambda / \nu$. Let $\operatorname{SRHT}(\lambda / \nu, \mu)$ be the set of special rim-hook tableaux of shape $\lambda / \nu$ and type $\mu$. Define the inverse Kostka numbers

$$
K_{\lambda, \mu}^{\prime}=\sum_{S \in \operatorname{SRHT}(\mu, \lambda)} \operatorname{sgn}(S)
$$

Theorem 6.1. For all $\lambda \in \operatorname{Par}_{N}$,

$$
m_{\lambda}\left(x_{1}, \ldots, x_{N}\right)=\sum_{\mu \in \operatorname{Par}_{N}} K_{\lambda, \mu}^{\prime} s_{\mu}\left(x_{1}, \ldots, x_{N}\right) .
$$

Proof. Multiplying by $a_{\delta(N)}$, it suffices to prove

$$
a_{\delta(N)} m_{\lambda}=\sum_{\mu \in \operatorname{Par}_{N}} \sum_{S \in \operatorname{SRHT}(\mu, \lambda)} \operatorname{sgn}(S) a_{\mu+\delta(N)} .
$$

The first step is to introduce an abacus interpretation for the product $a_{\delta(N)} m_{\lambda}$. A term in the polynomial $a_{\delta(N)}$ models a justified abacus containing $N$ beads labeled $w(N), \ldots, w(1)$ in positions $0, \ldots, N-1$ (respectively). Given such an abacus, we can view $m_{\lambda}\left(x_{1}, \ldots, x_{N}\right)$ as the sum of all distinct monomials $\prod_{i=1}^{N} x_{w(i)}^{e(i)}$ such that the exponent sequence $(e(1), \ldots, e(N))$ is a rearrangement of $\left(\lambda_{1}, \ldots, \lambda_{N}\right)$. (Here and below, we view elements of $\operatorname{Par}_{N}$ as partitions with exactly $N$ parts, some of which may be zero.) The multiplication of $a_{\delta(N)}$ by one of these monomials can be 
implemented on the abacus as follows. Imagine moving the $N$ justified beads from their current runner to a new, initially empty runner by moving each bead $w(i)$ from position $N-i$ on the old runner to position $N-i+e(i)$ on the new runner. Call such a transformation of the justified abacus a $\lambda$-move. A given $\lambda$-move either causes a bead collision on the new runner or else produces a new abacus (enumerated by a monomial in $a_{\mu+\delta(N)}\left(x_{1}, \ldots, x_{N}\right)$ for some $\left.\mu \in \operatorname{Par}_{N}\right)$.

First consider the situation where a bead collision occurs. Choose $i$ minimal such that bead $w(i)$ collides with some other bead on the new runner and then choose $j$ minimal such that bead $w(i)$ collides with $w(j)$. Create a new object counted by $a_{\delta(N)} m_{\lambda}$ by switching bead $w(i)$ and $w(j)$ on the old abacus and switching $e(i)$ and $e(j)$ in the exponent vector. This defines a sign-reversing, weight-preserving involution that cancels all objects in which bead collisions occur.

To complete the proof, we must find a sign-preserving, weight-preserving bijection $\phi$ from the set $X$ of uncanceled objects counted by $a_{\delta(N)} m_{\lambda}$ to the signed weighted set $\bigcup_{\mu \in \operatorname{Par}_{N}} \operatorname{SRHT}(\mu, \lambda) \times \operatorname{Abc}(\mu)$. For this purpose, let us fix $\mu \in \operatorname{Par}_{N}$ and consider the ways in which a justified abacus with $N$ beads can be transformed into an abacus in $\operatorname{Abc}(\mu)$ by means of a $\lambda$-move. Let us temporarily ignore bead labels and signs, concentrating at first only on the positions of the $N$ beads. The positions of the $N$ beads on the old runner are the entries in the sequence $\delta(N)=(N-1, N-$ $2, \ldots, 2,1,0)$. A $\lambda$-move adds some rearrangement of the sequence $\lambda=\left(\lambda_{1}, \ldots, \lambda_{N}\right)$ to the sequence $\delta(N)$. We will obtain an abacus in $\operatorname{Abc}(\mu)$ iff the sum of these sequences is some rearrangement of the sequence $\mu+\delta(N)=\left(\mu_{1}+N-1, \ldots, \mu_{N}+N-N\right)$.

We now show that the rearrangements of $\lambda$ that produce abaci in $\operatorname{Abc}(\mu)$ can be naturally encoded by special rim-hook tableaux of shape $\mu$ and type $\lambda$. The proof will use induction on $N$. Let us first illustrate the idea of the proof by considering an example. Take $N=5, \mu=(7,5,4,4,2)$, and $\lambda=(8,7,6,1,0)$. We seek rearrangements of the vector $(8,7,6,1,0)$ which, when added to the vector $(4,3,2,1,0)$, produce a rearrangement of $\mu+\delta(N)=(11,8,6,5,2)$. In this example, the only solution turns out to be $(1,8,0,7,6)+(4,3,2,1,0)=(5,11,2,8,6)$. We can visualize this solution using the following special rim-hook tableau, in which the ribbons (from top to bottom) have lengths $(1,8,0,7,6)$ :

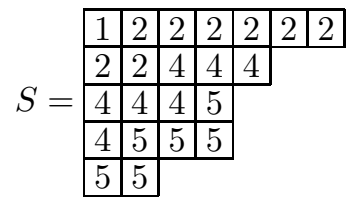

If we start with a labeled justified abacus $54321000 \cdots$ and perform a $\lambda$-move using the rearrangement $(1,8,0,7,6)$, we obtain the abacus $003001504002000 \cdots \in \operatorname{Abc}(\mu)$. The sign of this abacus, namely, $\operatorname{sgn}(31542)=-1$, differs from the sign of the original abacus, namely, $\operatorname{sgn}(54321)=+1$, by a factor of $(-1)^{5}=\operatorname{sgn}(S)$. A similar remark holds if the original abacus had involved some other permutation of the five labels.

With this example in mind, we return to the general proof. We are seeking permutations $j_{1} \cdots j_{N}$ and $k_{1} \cdots k_{N}$ satisfying the system of equations

$$
\begin{aligned}
0+\lambda_{j_{N}} & =\mu_{k_{N}}+N-k_{N}, \\
1+\lambda_{j_{N-1}}= & \mu_{k_{N-1}}+N-k_{N-1}, \\
\cdots & \cdots \\
N-1+\lambda_{j_{1}} & =\mu_{k_{1}}+N-k_{1} .
\end{aligned}
$$

In particular, to satisfy the first equation, we need an index $j=j_{N}$ and an index 
$k=k_{N}$ such that $\lambda_{j}=\mu_{k}+N-k$. If such an index exists, we encode it by drawing the unique border ribbon of length $\lambda_{j}$ starting in the leftmost cell of row $N$ of $\mu$. By choice of $j$ and $k$, this border ribbon must end in the rightmost cell of row $k$ of $\mu$. In terms of the abaci, the $\lambda$-move encoded by $j_{1} \cdots j_{N}$ moves the bead in position 0 on the old runner (the $N$ th bead from the right) to position $\mu_{k}+N-k$ on the new runner (which will become the $k$ th bead from the right). Thus this bead "moves past" $N-k$ other beads during the $\lambda$-move, which causes a sign change of $(-1)^{N-k}$ for any choice of labels. But $(-1)^{N-k}$ is precisely the sign of the border ribbon we just drew.

To finish solving system (6.1), let $\lambda^{*}$ be the partition obtained by dropping one part $\lambda_{j}$ from $\lambda$, and let $\mu^{*}$ be the partition in $\operatorname{Par}_{N-1}$ obtained by erasing the cells of $\mu$ occupied by the ribbon that starts in row $N$. Suppose we ignore the first equation in the system (6.1) and subtract 1 from both sides of the remaining $N-1$ equations. One may check that the resulting system of $N-1$ equations is precisely the system we must solve to change a justified abacus to an $(N-1)$-bead abacus in $\mathrm{Abc}\left(\mu^{*}\right)$ by means of a $\lambda^{*}$-move. (For instance, in the example considered earlier, after we move a bead from position 0 to position 6 (accounting for the lowest rim-hook in the displayed tableau), we have $\lambda^{*}=(8,7,1,0)$ and $\mu^{*}=(7,5,3,1)$. Having moved one bead, we are left with the task of moving beads from positions $(4,3,2,1)=(1,1,1,1)+\delta(4)$ to positions $(11,8,5,2)=(1,1,1,1)+\mu^{*}+\delta(4)$ using the moves in $\lambda^{*}=(8,7,1,0)$. $)$ By induction on $N$, the solutions of the reduced system are encoded by special rim-hook tableaux $S^{*}$ of shape $\mu^{*}$ and type $\lambda^{*}$; furthermore, the net sign change going from the old abacus to the new abacus (disregarding the bead originally in position 0 ) is $\operatorname{sgn}\left(S^{*}\right)$. It follows that all solutions of the original system are encoded by special rim-hook tableaux $S$ of shape $\mu$ and type $\lambda$; furthermore, the net sign change going from the old abacus to the new abacus (taking all beads into account) is $\operatorname{sgn}(S)$.

The preceding discussion contains an implicit recursive definition of the desired bijection $\phi$. More explicitly, suppose $z=(w(N) \cdots w(1) 000 \cdots, e(N) \cdots e(1)) \in X$ is an uncanceled object counted by $a_{\delta(N)} m_{\lambda}$. Then $\phi(z)=(S, v)$, where $v$ is the abacus obtained from the first component of $z$ by moving bead $w(i)$ right $e(i)$ positions for all $i$ and $S$ is the unique special rim-hook tableau of shape $\mu=\operatorname{sh}(v)$ that has a rim-hook of length $e(i)$ starting in the leftmost cell of row $i$ of the diagram. The preceding arguments show that $\phi$ preserves signs and weights. To compute $\phi^{-1}(S, v)$, it suffices to note that the sequence $(e(1), \ldots, e(N))$ is the content of the rim-hook tableau $S$. Knowledge of this sequence allows us to reverse the $\lambda$-move and recover $w(N) \cdots w(1)$. Thus, $\phi$ is a bijection.

The proof extends, mutatis mutandis, to describe the fixed points of $\lambda$-moves on abaci that are not necessarily justified. We thereby deduce the following Pieri-like rule for multiplying a Schur polynomial by a monomial symmetric polynomial.

Theorem 6.2. For all $\nu, \lambda \in \operatorname{Par}_{N}$,

$$
s_{\nu}\left(x_{1}, \ldots, x_{N}\right) m_{\lambda}\left(x_{1}, \ldots, x_{N}\right)=\sum_{\mu \in \operatorname{Par}_{N}} K_{\lambda, \mu / \nu}^{\prime} s_{\mu}\left(x_{1}, \ldots, x_{N}\right)
$$

where $K_{\lambda, \mu / \nu}^{\prime}=\sum_{S \in \operatorname{SRHT}(\mu / \nu, \lambda)} \operatorname{sgn}(S)$.

Example 9. Take $N=6, \nu=(3,3,2,0,0,0)$, and $\lambda=(4,4,3,2,0,0)$. We can start with the abacus $v=65400302100 \cdots$ counted by $a_{\nu+\delta(6)}$ and do a $\lambda$-move to obtain the abacus $v^{*}=0045600013200 \cdots$ of shape $\mu=(5,5,5,2,2,2)$. In this $\lambda$ move, beads $(6,5,4,3,2,1)$ move $(4,2,0,4,3,0)$ positions, respectively. This $\lambda$-move 
is modeled by the following special rim-hook tableau of shape $\mu / \nu$ and type $\lambda$ :

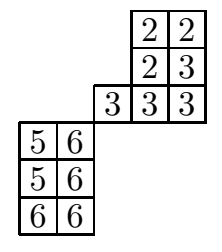

\section{REFERENCES}

[1] D. Beck, J. Remmel, And T. Whitehead, The combinatorics of transition matrices between the bases of symmetric functions and the $B_{n}$ analogues, Discrete Math., 153 (1996), pp. 327.

[2] E. Bender And D. Knuth, Enumeration of plane partitions, J. Combin. Theory Ser. A, 13 (1972), pp. 40-54.

[3] R. Borcherds, Modular Forms and Infinite Products, Rademacher lectures at the University of Pennsylvania, Philadelphia, PA, 2003.

[4] J. Carbonara, A. Kulikauskas, and J. Remmel, A combinatorial proof of the equivalence of the classical and combinatorial definitions of Schur functions, J. Combin. Theory Ser. A, 72 (1995), pp. 293-301.

[5] Ö. EĞEcioĞLu And J. Remmel, A combinatorial interpretation of the inverse Kostka matrix, Linear Multilinear Algebra, 26 (1990), pp. 59-84.

[6] W. Fulton, Young Tableaux, Cambridge University Press, Cambridge, 1997.

[7] G. James and A. Kerber, The Representation Theory of the Symmetric Group, AddisonWesley, Reading, MA, 1981.

[8] M. KASHIWARA, Crystallizing the q-analogue of universal enveloping algebras, Comm. Math. Phys., 133 (1991), pp. 249-260.

[9] M. Kashiwara, Crystallization of quantized enveloping algebras, Sugaku Expositiones, 7 (1994), pp. 99-115.

[10] I. G. Macdonald, Symmetric Functions and Hall Polynomials, 2nd ed., Oxford University Press, Oxford, 1995.

[11] J. Remmel And M. Shimozono, A simple proof of the Littlewood-Richardson rule and applications, Discrete Math., 193 (1998), pp. 257-266.

[12] R. Stanley, Enumerative Combinatorics, Vol. 2, Cambridge University Press, Cambridge, 1999.

Copyright (c) by SIAM. Unauthorized reproduction of this article is prohibited. 